\title{
Proceeding
}

8th INSHS International Christmas Sport Scientific Conference, 5-7 December 2013. International Network of Sport and Health

Science. Szombathely, Hungary

\section{Changes to eating habits of young men after finishing a diving course}

\author{
JANA JUŘÍKOVÁ \\ Faculty of Sports Studies, Department of Kinesiology, Masaryk University, Brno, Czech Republic
}

\begin{abstract}
Juříková, J. (2014). Changes to eating habits of young men after finishing a diving course. J. Hum. Sport Exerc., 9(Proc1), pp.S449-S459. At the Military Academy Vyškov in the Czech Republic there are diving courses arranged for soldiers. The courses take two months. The young men coming to the courses have different eating habits that they have gained in the family and/or in the military crew. A diving training is very hard, both physically and mentally. Increased energy expenditure requires also an adequate energy dietary intake.Mlt was investigated whether eating habits of soldiers have changed. The eating habits were investigated by anonymous questionnaire before opening a course and after finishing a course. Also anthropometric parameters were measured before opening and after finishing a course. It was measured: body weight, body height and body mass index that was calculated from the previous data. The physical activity of diving course participants in leisure time was identified too. The results demonstrated that the diet composition was radically changed for all investigated soldiers after finishing a diving course. The percentage of soldiers who eat for breakfast meat, dairy products, fruit and vegetables has increased. At the same time the consumption of bakery products has decreased. For lunch the consumption of meat, fat foods, potatoes, cereals orders, fruit and vegetables has increased. For dinner the consumption of meat products, fat foods, dairy products, potatoes, cereals orders, fruit and sweets has increased.After finishing a diving course the consumption of alcohol was slightly increased, participants of a diving course drank mainly beer. The diving training had no influence on smoking of cigarettes. Key words: SOLDIERS, DIET, BREAKFAST, LUNCH, DINNER.
\end{abstract}

Corresponding author. Faculty of Sports Studies, Department of Kinesiology, Masaryk University, Brno, Czech Republic.

E-mail: jurikova@fsps.muni.cz

8th INSHS International Christmas Sport Scientific Conference, 5-7 December 2013. International Network of Sport and Health Science. Szombathely, Hungary.

JOURNAL OF HUMAN SPORT \& EXERCISE ISSN 1988-5202

(c) Faculty of Education. University of Alicante

doi:10.14198/jhse.2014.9.Proc1.32 


\section{INTRODUCTION}

The Military Academy in Vyškov is one of the best equipped working places of the Czech Army regarding not only the military training but also sports activities of the soldiers. The premises of the Academy includes a running track, sports hall, tankodrome, shooting range, combat simulators and other modern technical equipment. It is the only place in the Czech Republic which offers indoor training hall with a pool to train driving tanks under the water. It can also be used to train divers in difficult conditions. Soldiers who are interested in diving can enroll into a diving course which will train them in precision swimming and diving in a large sports pool. Part of the course is also training divers in a tank ford and in the water diving under the ice in natural conditions. It is very demanding to pass the course and by no means that it could be compared with hobby diving courses. More often than not it happens that not all participants finish the course.

In the past when the military service was compulsory in the Czech Republic, only soldiers under military training could enroll into the course. Nowadays, the course is open to the general public, but not many civilians are interested in it due to the difficulty and smaller attractiveness if compared with hobby sea diving. Thus, these courses are still attended by the soldiers only.

Soldiers who enter the course have their own eating habits which originated at their homes or in their army units at the time when they were not performing so demanding activities. The aim of this paper is to find out whether usual daily portions of food which is allotted in the barracks are sufficient, or whether the eating habits of the soldiers have changed and they have to buy more food or drinks with their own money.

\section{MATERIALS AND METHODOLOGY}

The courses which the soldiers enrolled in lasted for two months. Soldiers who had been selected to take part in the courses arrived from their units and they stayed in the barracks of the Vyškov unit during the whole course. They had their meals in unit's canteen and they were allowed to use both the sports equipment of the unit and of the Military Academy. They can choose their meal from 4 menus of lunch and from 2 menus of dinner and 1 snack. To fill all courses, a certain number of places were offered to soldiers of all army units of the Czech Republic. Either exactly the offered number or a smaller number of soldiers applied for the courses, therefore everyone who applied was accepted; there was no subsequent selection procedure. The only condition to enter the course was to know how to swim which did not seem to be a problem at first. Later, it was revealed that though all participants could swim their swimming technique was frequently on a very low level, therefore the beginning of the course was devoted to improving the technique of all swimming styles. When all the participants became very good swimmers, they could also become good divers. This stage of the diving training was taken by all participants. However, diving was much more demanding: after only a few lessons of diving in the pool, the divers were trained in a flooded tank, in tank ford and in rivers and lakes. This part of the diving course was very physically and psychically demanding and it happened that several participants left the course at this stage and they were not able to finish the course. Diving under the ice in the water was evaluated as the most difficult by soldiers.

The soldiers in the Czech Army are allotted different portions of food according to the difficulty of their work. These portions are divided into:

1. Basic food portion

2. Food portion for students at military secondary schools

3. Food portion for pilots 
4. Food portion for paratroopers (Defense Ministry Notice No. 266/1999 Coll, 1999) (Defense Ministry Notice No. 269/1999 Coll, 1999.

Soldiers under basic military training who take part in the diving course are included in the Basic food portion. Basic food portion is provided to a soldier who does not have the right for a different food portion. A duty officer who performs the function for at least 24 hours at military schools and in air and paratroop units, which do not allot Basic food portion, gets such food portions, which is commonly allotted in the respective unit.

There are also additions to the individual food portions according to the difficulty of performed service; they include:

a) A food addition - health

b) B food addition - demanding service

c) C food addition - especially demanding service

d) D food addition - continuous military training

e) $\quad$ E food addition - during sports activities

f) $\quad F$ food addition - during sports competitions within the scope of Defense Ministry

g) G food addition - for pilots in service on the days of flying activities

h) $\quad \mathrm{H}$ food addition - for pilots in service who are members of units of supersonic and subsonic airplanes equipped with weapon systems and transport planes (Defense Ministry Notice No. 266/1999 Coll, 1999)

The above food additions are allotted to professional soldiers, pilots and paratroopers (Defense Ministry Notice No. 269/1999 Coll, 1999).

Even though it appears that soldiers in the diving course should have the right for at least the $E$ food addition - sports activities - for the duration of the course, or B or C food additions - demanding service or especially demanding service - they are not entitled to any addition. Despite having performed several researches concerning this issue, e.g. by Březina et al. (2000) and Březina et al. (2002) and despite having sent an appeal to the Defense Ministry based on the findings of the researches to provide food additions for the divers during the diving course, no additions were allotted to the divers.

Researching eating habits. The eating habits of the soldiers in the diving course were researched with a questionnaire. A part of a standardized questionnaire created at the Faculty of Medicine of Masaryk University in Brno (Czech Republic) by Brázdová \& Fiala (1998) was used for the questionnaire. The questionnaire was presented to the soldiers twice: first before starting the diving course and then after finishing it. The questionnaire was anonymous. All questions were explained to the soldiers in advance, which resulted in submitting all questionnaires with complete answers and without mistakes that could be caused by misunderstanding. Thus, the return rate of the questionnaires was both before starting the course and after finishing it $100 \%$. Before and after the course, the participants were measured for anthropometric indicators such as body weight and height and these values were used to calculate the Body Mass Index (BMI).

Measuring body weight

Body weight was measured on medical scales by Soenhle Company with precision of $0.1 \mathrm{~kg}$. During weighting, the examined person was wearing only underwear and no shoes (Kleinwachterová \& Brázdová, 1992). The weight of the underwear was not considered. 
Measuring body height

Body height was measured with an anthropometer. The anthropometer was a part of the medical digital scales with precision of $0.1 \mathrm{~mm}$. During measuring body weight and height, the examined person was standing in erect stand, heels together, the arms along the body and erect head.

Calculating the Body Mass Index (BMI)

The Body Mass Index (usually abbreviated to BMI) is a secondary indicator which is derived from body weight and height. Measuring BMl is widely used both by civilians and in the army (Juřiková et al., 2001). The measured values of body weight and height were used to calculate BMl using the following formula

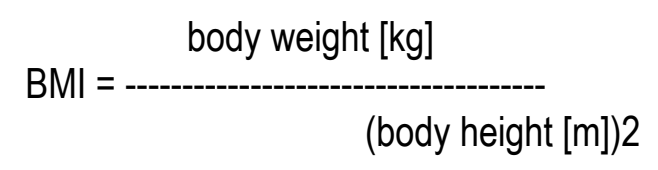

Obesity classification is presented in Table 1.

Participants of the research

All participants of the diving course were included in the research. The course took place between February and April 2000. Altogether 36 soldiers enrolled in the course out of whose 32 finished the course. This works present anthropometric indicators and eating habits only of the soldiers who finished the course. The table below shows anthropometric indicators before and after the diving course as well as the age of the researched soldiers.

Table 1. BMI categories, obesity classification (according to WHO) and its relationship to health risks (Gauner, 2001)

\begin{tabular}{lll}
\hline \multicolumn{1}{c}{ BMI } & Category by WHO & \multicolumn{1}{c}{ Health risks } \\
\hline$\leq 18,5$ & underweight & malnutrition, anorexia \\
$18,5-24,9$ & normal weigh & minimal \\
$25,0-29,9$ & overweight & slightly increased \\
$30,0-34,9$ & $1^{\text {st }}$ degree of obesity & moderately high \\
$35,0-39,9$ & $2^{\text {nd }}$ degree of obesity & high \\
$\geq 40$ & $3^{\text {rd }}$ degree of obesity & very high \\
\hline
\end{tabular}

Table 2. Shows the body physique of men according to BMI

\begin{tabular}{ll}
\hline BMI & Men \\
\hline$<18$ & skinny \\
$18,0-23,0$ & slim \\
$23,1-25,0$ & adequate \\
$25,1-30,0$ & robust \\
$>30$ & obese \\
\hline
\end{tabular}


Juřiková / Changes to eating habits of young men after finishing a diving course JOURNAL OF HUMAN SPORT \& EXERCISE

Table 3. Body physique of men according to BMI (Kleinwachterová \& Brázdová, 1992)

\begin{tabular}{|c|c|c|c|c|c|c|c|}
\hline $\begin{array}{c}\text { Number of } \\
\text { person }\end{array}$ & $\begin{array}{c}\text { Body } \\
\text { weight } \\
\text { behind } \\
{[\mathrm{kg}]}\end{array}$ & $\begin{array}{l}\text { Body weight } \\
\text { after [kg] }\end{array}$ & $\begin{array}{l}\text { Change of } \\
\text { the body } \\
\text { weight [kg] }\end{array}$ & $\begin{array}{c}\text { BMI } \\
\text { behind }\end{array}$ & $\begin{array}{l}\text { BMI } \\
\text { after }\end{array}$ & $\begin{array}{l}\text { Change } \\
\text { od BMI }\end{array}$ & Age [years] \\
\hline 1 & 66.9 & 64.3 & -2.6 & 22.2 & 21.4 & -0.8 & 21 \\
\hline 2 & 71.0 & 70.1 & -0.9 & 21.2 & 20.9 & -0.3 & 19 \\
\hline 3 & 72.5 & 69.9 & -2.6 & 23.0 & 22.2 & -0.8 & 22 \\
\hline 4 & 86.5 & 81.9 & -4.6 & 26.8 & 25.4 & -1.4 & 20 \\
\hline 5 & 83.9 & 83.4 & -0.5 & 28.0 & 27.9 & -0.1 & 28 \\
\hline 6 & 72.0 & 69.9 & -2.1 & 24.5 & 23.8 & -0.7 & 26 \\
\hline 7 & 74.6 & 71.7 & -2.9 & 22.9 & 22.0 & -0.9 & 27 \\
\hline 8 & 79.2 & 86.9 & 7.7 & 25.1 & 27.6 & 2.5 & 26 \\
\hline 9 & 85.1 & 86.9 & 1.8 & 27.3 & 27.9 & 0.6 & 26 \\
\hline 10 & 79.7 & 77.0 & -2.7 & 25.3 & 24.4 & -0.9 & 28 \\
\hline 11 & 84.6 & 83.0 & -1.6 & 26.6 & 26.0 & -0.6 & 25 \\
\hline 12 & 71.9 & 70.3 & -1.6 & 24.7 & 24.2 & -0.5 & 21 \\
\hline 13 & 66.9 & 67.2 & 0.3 & 21.5 & 21.6 & 0.1 & 18 \\
\hline 14 & 78.2 & 76.4 & -1.8 & 26.3 & 25.7 & -0.6 & 20 \\
\hline 15 & 59.2 & 60.6 & 1.4 & 19.7 & 20.1 & 0.4 & 19 \\
\hline 16 & 51.0 & 60.1 & 9.1 & 20.8 & 28.6 & 7.8 & 20 \\
\hline 17 & 72.1 & 70.1 & -2.0 & 22.2 & 21.6 & -0.6 & 19 \\
\hline 18 & 81.0 & 79.3 & -1.7 & 27.1 & 26.5 & -0.6 & 22 \\
\hline 19 & 73.8 & 72.9 & -0.9 & 24.0 & 23.7 & -0.3 & 19 \\
\hline 20 & 68.0 & 69.2 & 1.2 & 21.3 & 21.7 & 0.4 & 19 \\
\hline 21 & 68.5 & 67.0 & -1.5 & 25.3 & 24.8 & -0.5 & 19 \\
\hline 22 & 69.9 & 71.1 & 1.2 & 20.5 & 20.9 & 0.4 & 19 \\
\hline 23 & 73.4 & 73.0 & -0.4 & 23.6 & 23.4 & -0.2 & 19 \\
\hline 24 & 76.3 & 79.6 & 3.3 & 21.7 & 22.6 & 0.9 & 19 \\
\hline 25 & 89.8 & 90.9 & 1.1 & 27.7 & 28.1 & 0.4 & 18 \\
\hline 26 & 68.0 & 69.1 & 1.1 & 20.5 & 20.9 & 0.4 & 23 \\
\hline 27 & 62.0 & 62.6 & 0.6 & 19.7 & 19.9 & 0.2 & 19 \\
\hline 28 & 69.7 & 71.6 & 1.9 & 21.8 & 22.3 & 0.5 & 19 \\
\hline 29 & 66.9 & 68.3 & 1.4 & 23.4 & 23.9 & 0.5 & 18 \\
\hline 30 & 88.8 & 86.7 & -2.1 & 24.1 & 23.5 & -0.6 & 19 \\
\hline 31 & 73.6 & 70.8 & -2.8 & 25.3 & 24.3 & -1.0 & 22 \\
\hline 32 & 62.1 & 64.7 & 2.6 & 20.3 & 21.1 & 0.8 & 18 \\
\hline
\end{tabular}


Table 4. The age and anthropometric indicator of the soldiers before and after the diving course

\begin{tabular}{|c|c|c|}
\hline $\begin{array}{l}\text { Number } \\
\text { of person }\end{array}$ & Breakfast behind diving course & Breakfast after diving course \\
\hline 1 & pastry, meat products & meat, dairy products \\
\hline 2 & pastry, dairy products & pastry, meat \\
\hline 3 & pastry, fat products & pastry, meat products \\
\hline 4 & meat, dairy products, vegetables & $\begin{array}{l}\text { dairy products, meat products, fat food, fruit, } \\
\text { vegetables, sweets }\end{array}$ \\
\hline 5 & pastry, fruit & pastry, meat, fruit, vegetables \\
\hline 6 & pastry, fruit & pastry, dairy products, sweets \\
\hline 7 & pastry, fruit, vegetables & dairy products, fruit, vegetables \\
\hline 8 & pastry, dairy products, fruit & pastry, dairy products, fruit \\
\hline 9 & pastry, dairy products & $\begin{array}{l}\text { pastry, dairy products, meat, meat products, } \\
\text { fruit, vegetables }\end{array}$ \\
\hline 10 & fruit, dairy products, vegetables & dairy products, meat, potatoes, fruit \\
\hline 11 & pastry, dairy products, meat products, fats & pastry, fats \\
\hline 12 & pastry, fruit, fats & pastry, dairy products, meat products, fats \\
\hline 13 & pastry, fats & pastry, dairy products \\
\hline 14 & pastry, fats & $\begin{array}{l}\text { pastry, dairy products, meat, meat products, } \\
\text { sweets }\end{array}$ \\
\hline 15 & pastry, dairy products & pastry, meat products \\
\hline 16 & pastry, fats & pastry, dairy products, sweets \\
\hline 17 & dairy products, fruit, vegetables & meat products, fats, sweets \\
\hline 18 & pastry, fats, fruit & pastry, dairy products \\
\hline 19 & pastry, meat products, fruit & $\begin{array}{l}\text { pastry, meat, potatoes, dairy products, meat } \\
\text { products, fruit }\end{array}$ \\
\hline 20 & pastry, meat, meat products, fats & pastry, fats, sweets \\
\hline 21 & pastry, fats, fruit & pastry, meat, meat products \\
\hline 22 & pastry, dairy products, meat products, sweets & pastry, meat products \\
\hline 23 & pastry, dairy products, meat products & pastry, meat \\
\hline 24 & pastry, fats & pastry, meat, vegetables \\
\hline 25 & pastry, fats, fruit, vegetables & pastry, dairy products, meat, meat products \\
\hline 26 & pastry, meat, fats & pastry, meat, fats \\
\hline 27 & pastry, sweets & pastry, sweets \\
\hline 28 & pastry, sweets & pastry, meat products \\
\hline 29 & pastry, sweets & pastry, dairy products, vegetables, sweets \\
\hline 30 & pastry, sweets & dairy products, fats, meat products, fruits \\
\hline 31 & pastry, sweets & pastry, sweets \\
\hline 32 & pastry, fats, sweets & pastry, meat products, fats \\
\hline
\end{tabular}

\section{RESULTS AND DISCUSSION}

The diving course was attended by 32 soldiers between the ages of 18 and 28 . The average age was 21.2 $( \pm 3.2)$. As can be seen in Table 3,18 soldiers lost weight during the course; the weight loss ranged from 0.4 to $4.6 \mathrm{~kg}$. 14 soldiers put on weight, from 0.3 to $7.7 \mathrm{~kg}$. This change in their body weight was caused by 
hard training and also by a change in their eating habits. The changes in their eating habits are presented in Table 4 - 7.

Table 5. Kind of food eaten by researched soldiers mainly for breakfast (before and after the diving course)

\begin{tabular}{|c|c|c|}
\hline $\begin{array}{l}\text { Number } \\
\text { of person }\end{array}$ & $\begin{array}{l}\text { Lunch behind diving } \\
\text { course }\end{array}$ & Lunch after diving course \\
\hline 1 & meat, potatoes & meat, potatoes, cereals, fruit, dairy products \\
\hline 2 & $\begin{array}{l}\text { meat, potatoes, cereals, } \\
\text { vegetables }\end{array}$ & $\begin{array}{l}\text { meat, potatoes, cereals, vegetables, fruit, meat products, fat } \\
\text { products, dairy products, sweets }\end{array}$ \\
\hline 3 & meat, cereals & meat, potatoes \\
\hline 4 & $\begin{array}{l}\text { meat, } \quad \text { potatoes, } \\
\text { vegetables }\end{array}$ & potatoes, cereals, vegetables, fruit, sweets \\
\hline 5 & potatoes, vegetables, fruit & meat, potatoes, cereals, vegetables, fruit \\
\hline 6 & meat, potatoes, cereals & meat, cereals, fat products \\
\hline 7 & potatoes, vegetables & meat, potatoes, cereals, vegetables \\
\hline 8 & meat, cereals & meat, cereals \\
\hline 9 & $\begin{array}{l}\text { meat, cereals, vegetables, } \\
\text { fruit }\end{array}$ & $\begin{array}{l}\text { meat, potatoes, cereals, vegetables, fruit, meat products, dairy } \\
\text { products }\end{array}$ \\
\hline 10 & cereals, fruit & meat, potatoes, cereals, vegetables, fruit \\
\hline 11 & $\begin{array}{l}\text { meat, potatoes, cereals, } \\
\text { fats }\end{array}$ & meat, fats, sweets \\
\hline 12 & meat, vegetables & meat, potatoes, cereals \\
\hline 13 & meat, potatoes & potatoes, cereals, meat products \\
\hline 14 & meat, potatoes, cereals & meat, potatoes \\
\hline 15 & meat, potatoes, cereals & meat, potatoes, cereals \\
\hline 16 & meat, potatoes & meat, potatoes, cereals \\
\hline 17 & $\begin{array}{l}\text { potatoes, } \quad \text { cereals, } \\
\text { vegetables }\end{array}$ & potatoes, fruit \\
\hline 18 & meat, vegetables, fruit & potatoes, fruit \\
\hline 19 & meat, potatoes, fruit & meat, cereals \\
\hline 20 & $\begin{array}{l}\text { meat, } \quad \text { potatoes, } \\
\text { vegetables }\end{array}$ & meat, cereals \\
\hline 21 & $\begin{array}{l}\text { meat, potatoes, cereals, } \\
\text { fruit, sweets }\end{array}$ & meat, potatoes, cereals \\
\hline 22 & meat, cereals & meat, potatoes, fruit \\
\hline 23 & meat, fats & potatoes, cereals, meat products \\
\hline 24 & $\begin{array}{l}\text { meat, potatoes, cereals, } \\
\text { meat products, fats }\end{array}$ & meat, vegetables \\
\hline 25 & meat, potatoes & $\begin{array}{l}\text { meat, potatoes, vegetables, fruit, meat products, fats, dairy } \\
\text { products, sweets }\end{array}$ \\
\hline 26 & $\begin{array}{l}\text { meat, potatoes, cereals, } \\
\text { meat products, fruit }\end{array}$ & meat, cereals \\
\hline 27 & meat, cereals & cereals, sweets \\
\hline 28 & meat, cereals & potatoes, cereals, vegetables, meat products \\
\hline 29 & $\begin{array}{l}\text { meat, potatoes, meat } \\
\text { products }\end{array}$ & meat, potatoes, cereals, meat products \\
\hline
\end{tabular}


30 meat, sweets

31 meat, cereals, sweets

32 meat, potatoes cereals, fats, sweets

meat, potatoes

meat, potatoes, cereals, fruit

Table 5 reveals that originally the most frequent food for breakfast was pastry, fruit, dairy products and fats. After finishing the diving course, the consumption of pastry lowered, even though pastry still prevailed in the composition of breakfast. The consumption of fats and fruit was also smaller while consumption of dairy products, meat and vegetables increased. Before starting the course, only one soldier stated that he often eats meat for breakfast while after finishing the course, the number of soldiers eating meat for breakfast increased to ten, which makes nearly one third of questioned persons. There was also increase in the number of soldiers who ate sweets after breakfast.

Table 6 shows that the composition of lunch did not change much during the course. Both before the course and after it, the soldiers were eating mainly lunch with potatoes or cereals. Increased consumption of meat and dairy products for lunch is a proof that after lunch the soldiers were some additional meat. Frequently, they were also buying yoghurts and other dairy products and if compared to the time before the diving course, more soldiers started to eat sweets after lunch. This information reveals that the diet composition of their lunch was not sufficient for the soldiers in the diving course.

Table 6. Kind of food eaten by researched soldiers mainly for lunch (before and after the diving course).

\begin{tabular}{|c|c|c|}
\hline $\begin{array}{l}\text { Number } \\
\text { of } \\
\text { person }\end{array}$ & Dinner behind diving course & Dinner after diving course \\
\hline 1 & meat, potatoes, pastry & meat, potatoes, pastry \\
\hline 2 & potatoes, pastry & $\begin{array}{l}\text { meat, potatoes, cereals, vegetables, fruit, meat } \\
\text { products, fat products, dairy products, sweets }\end{array}$ \\
\hline 3 & $\begin{array}{l}\text { meat, meat products, pastry, dairy products, } \\
\text { fruit }\end{array}$ & meat, potatoes, fruit \\
\hline $\begin{array}{l}4 \\
5\end{array}$ & $\begin{array}{l}\text { meat, potatoes } \\
\text { potaotes, cereals, vegetables, fruit }\end{array}$ & $\begin{array}{l}\text { meat, meat products, pastry, fruit, sweets } \\
\text { meat, meat products, potatoes, pastry, } \\
\text { vegetables, fruit }\end{array}$ \\
\hline 6 & $\begin{array}{l}\text { meat, meat products, vegetables, fruit, } \\
\text { sweets }\end{array}$ & $\begin{array}{l}\text { meat, meat products, dairy products, pastry, fat } \\
\text { products }\end{array}$ \\
\hline 7 & potatoes, vegetables, pastry & $\begin{array}{l}\text { potatoes, dairy products, pastry, vegetables, } \\
\text { fruit }\end{array}$ \\
\hline $\begin{array}{l}8 \\
9\end{array}$ & $\begin{array}{l}\text { pastry, vegetables, fruit } \\
\text { pastry, dairy products, vegetables, fruit }\end{array}$ & $\begin{array}{l}\text { meat, pastry, fruit } \\
\text { meat, potatoes, meat products, pastry, dairy } \\
\text { products, vegetables, fruit, sweets }\end{array}$ \\
\hline 10 & pastry, fruit & $\begin{array}{l}\text { meat, potatoes, dairy products, pastry, } \\
\text { vegetables, fruit }\end{array}$ \\
\hline 11 & pastry, meat products & meat, potatoes, fats, pastry \\
\hline 12 & pastry, meat products & meat, potaotes, meat products, pastry \\
\hline 13 & pastry, meat products & potatoes, meat products, pastry \\
\hline 14 & meat, potatoes, pastry & meat, potatoes, pastry, fruit \\
\hline 15 & meat, potatoes, pastry, vegetables & meat, potatoes, pastry \\
\hline 16 & meat, potatoes & meat, vegetables, dairy products \\
\hline
\end{tabular}




\begin{tabular}{lll}
\hline 17 & dairy products, meat products, fats & fats, meat products, potatoes, sweets \\
18 & pastry, meat products & meat, potatoes, meat products, pastry \\
19 & meat, potatoes, meat products, pastry & meat, meat products, pastry \\
20 & potatoes, pastry, vegetables & meat products, potatoes, pastry \\
21 & meat, pastry, dairy products, fruit, sweets & meat, potatoes, pastry \\
22 & meat, pastry & meat products, pastry \\
23 & meat producst, meat, sweets & meat, potatoes, fats \\
24 & & meat, potatoes, meat products, pastry, \\
& meat, pastry, meat products, fats & vegetables \\
25 & meat, meat products, pastry, dairy products, & \\
& fruit & potatoes, pastry, vegetables, fruit \\
26 & meat, pastry & meat, pastry \\
27 & meat, pastry, sweets & meat, pastry, fats, vegetables, fruit, sweets \\
28 & meat, pastry, sweets & meat, potatoes, meat products, pastry \\
29 & pastry, meat products & meat products, potatoes, pastry \\
30 & meat, potatoes & fats, sweets \\
31 & pastry, sweets & x \\
32 & pastry, meat products, fats & meat products, potatoes, pastry \\
\hline
\end{tabular}

Table 7. Kind of food eaten by researched soldiers mainly for dinner (before and after the diving course).

\begin{tabular}{|c|c|c|}
\hline $\begin{array}{l}\text { Number of } \\
\text { person }\end{array}$ & Snacks behind diving course & Snacks after diving course \\
\hline 1 & dairy products & dairy products, meat, fruit, vegetables \\
\hline 2 & pastry, dairy products, meat products & $\begin{array}{l}\text { dairy products, meat, meat products, pastry, } \\
\text { fruit, vegetables, sweets }\end{array}$ \\
\hline 3 & fruit, vegetables & dairy products, fat products, fruit, sweets \\
\hline 4 & meat products & meat, meat products \\
\hline 5 & fruit, vegetables & fruit, sweets \\
\hline 6 & pastry, vegetables & $\begin{array}{l}\text { dairy products, meat products, pastry, } \\
\text { vegetables }\end{array}$ \\
\hline 7 & fruit, vegetables & fruit, vegetables \\
\hline 8 & pastry, fruit & dairy products, meat, pastry, fruit, vegetables \\
\hline 9 & pastry, fruit, vegetables & all \\
\hline 10 & $x$ & dairy products, fruit \\
\hline 11 & pastry, fats & fruit, sweets \\
\hline 12 & pastry, sweets & fats, pastry, sweets \\
\hline 13 & $\begin{array}{l}\text { dairy products, meat products, fats, fruit, } \\
\text { vegetables }\end{array}$ & meat, fruit \\
\hline 14 & $x$ & meat, pastry \\
\hline 15 & fruit, sweets & $\mathrm{x}$ \\
\hline 16 & $\begin{array}{l}\text { meat products, pastry, fruit, vegetables, } \\
\text { sweets }\end{array}$ & dairy products, fruit, vegetables, sweets \\
\hline 17 & dairy products, fruit, vegetables & $\begin{array}{l}\text { dairy products, meat products, fats, fruit, } \\
\text { vegetables, sweets }\end{array}$ \\
\hline 18 & pastry, fruit, vegetables & meat products, pastry, fruit \\
\hline
\end{tabular}




\begin{tabular}{|c|c|c|}
\hline 19 & $x$ & dairy products, fruit, sweets \\
\hline 20 & meat products, pastry & meat products, pastry, fats, sweets \\
\hline 21 & $\mathrm{x}$ & pastry, sweets \\
\hline 22 & pastry, vegetables & meat products, pastry \\
\hline 23 & meat products, pastry, sweets & meat, meat products, sweets \\
\hline 24 & $\begin{array}{l}\text { dairy products, meat products, pastry, } \\
\text { fruit, sweets }\end{array}$ & pastry, sweets \\
\hline 25 & dairy products, fruit, sweets & dairy products, sweets \\
\hline 26 & fats, sweets & pastry, sweets \\
\hline 27 & $\begin{array}{l}\text { dairy products, meets products, fruit, } \\
\text { sweets }\end{array}$ & pastry, sweets \\
\hline 28 & dairy products, sweets & $x$ \\
\hline 29 & meat, meat products & $x$ \\
\hline 30 & dairy products, sweets & pastry, potatoes \\
\hline 31 & pastry, sweets & $x$ \\
\hline 32 & fats, pastry, fruit & $x$ \\
\hline
\end{tabular}

As can be seen in Table 7, before starting the diving course, the soldiers were mainly eating meat and cereal sides for dinner. If they had just supper, they ate mainly products made of meat and pastry. After finishing the course, they preferred dinner to supper and they were eating mainly meat with potatoes and cereals. It is interesting that after dinner, smaller number of soldiers ate sweets than before the diving course. Before the course, no soldier stated that he would be eating fat food. After the course, two soldiers stated that they eat fat food both for lunch and dinner. One soldier stated that during the diving course he stopped going for dinners in the barracks and he started to buy food for his own money. The soldiers were not offered snacks during the diving course, however there was a possibility for them to buy some snack in a nearby canteen. Before starting the course, 28 soldiers were buying snack (87.5\%), after finishing the course it was by one soldier less. For snacks, they were mainly eating pastry, dairy products, fruit, vegetable and sweets. After finishing the course, there was a decrease in the number of soldiers who ate vegetable for snacks, on the other hand, the number of soldiers who were buying sweets increased, which show that the energetic need of their organisms grew during the diving course. The number of soldiers who ate dairy products and fats for snacks did not change. There was also increase in the number of soldiers who were eating meat. No soldier stated that he would be eating meat or fat food before starting the diving course; after finishing the course, seven soldiers stated that they eat meat for snacks and two soldiers that they eat mainly fat food for snacks.

During the course, there appeared a change in the body weight of the soldiers as well as in the redistribution of fat into hypodermis. Their BMI changed as well, which is shown in Table 3. Based on BMI, 15 soldiers (45.9\%) were included in the "slim" group before starting the diving course; after finishing it, it was only $14(43.8 \%)$, one soldier was moved to the "satisfactory" category due to increased BMI. Altogether, there were 6 soldiers $(18.8 \%)$ in the "satisfactory" category before the course, after the course it was 9 soldiers $(28.1 \%) .11$ soldiers $(34.4 \%)$ were "robust" before the diving course, after finishing the course, this category included only 9 soldiers $(28.1 \%)$. No soldier was overweight both before and after the diving course. In case of diving training during which BMI increases because fat is deposited in hypodermis as a reaction of the organism to staying in cold water, BMI categories such as obesity or severe obesity could not be used. This is the case also in other strength sports like weightlifting or wrestling etc. 


\section{CONCLUSIONS}

The results of the research show that during the diving training, the weight of the soldiers changed, which resulted also in the change of BMI: 3 soldiers (9.4\%) were moved to a group with lower BMI, 1 soldier (3.1 $\%$ ) was included in a group with higher BMI. Body weight of the majority of the soldiers decreased; this was observed in 18 soldiers (56.2 \%). Body weight of 14 soldiers (43.8\%) increased. As far as eating habits are concerned, it was revealed that during the course, the majority of the soldiers started to buy additional food apart from the food they were given in the barracks. The additional food included dairy products, meat and mainly sweets, which shows that their energetic expenditure was not sufficiently covered with the Basic Food Portion. Consumption of meat and dinners increased; the dinners were composed mainly from meat with cereals or potatoes.

\section{REFERENCES}

1. Brázdová, Z. \& Fiala, J. (1998). Dietary Guidelines in the Czech Republic . Compilation of papers of faculty od medicine in Brno No. 115. Brno, Czech republic: Faculty od Medicine of Masaryk University.

2. Březina, P., Juříková, J., Kreuziger, J. \& Šimůnek, J. (2002). A study to physiology of nutrition of members of Czech Army in relation to working and nutrition regime. The final report of Project of defense research. Vyškov, Czech republic: Military University of Ground Forces.

3. Březina, P., Šimůnek, J., Budíková, M., Juříková, J., Kreuziger, J., Jersák, R. \& Hurtík, T. (2000). The evaluation of physical aspects of the working regime and nutrition of selected groups of members of the Czech Army. The final report of the Project of defense research. Vyškov, Czech republic: Military University of Ground Forces.

4. Defense Ministry Notice NO. 266/1999 Coll. (1999). Praque, Czech republic: Defense Ministry.

5. Defense Ministry Notice NO. 269/1999 Coll. (1999). Prague, Czech republic: Defense Ministry.

6. Gauner, V. (2001). Obesity, minimum for practice. Prague: Triton.

7. Juř́ková, J., Komár, A. \& Kreuziger, J. (2001). BMI as a means to evaluation of adequate body weight and health. Military Professional, pp. $108-111$.

8. Kleinwachterová, H. \& Brázdová, Z. (1992). State of human nutrition and methods its inquire. Brno: Masaryk University. 